\title{
Association between COVID-19 vaccine hesitancy and generalized trust, depression, generalized anxiety, and fear of COVID-19
}

Yoichi Sekizawa ${ }^{*}$, Sora Hashimoto ${ }^{2}, K$ Kenzo Denda ${ }^{3}$, Sae Ochi ${ }^{4}$ and Mirai So ${ }^{5}$

\begin{abstract}
Background: Although numerous studies have been published on the predictors of COVID-19 vaccine hesitancy, some possible predictors remain underexplored. In this study, we explored the associations of unwillingness and indecisiveness regarding COVID-19 vaccination with generalized trust, mental health conditions such as depression and generalized anxiety, and fear of COVID-19.

Methods: Data of wave 1 (from October 27 till November 6, 2020) and wave 3 (from April 23 till May 6, 2021) of a longitudinal online study conducted in Japan were used for the analyses. Unvaccinated participants were asked at wave 3 about their willingness to be vaccinated, with possible responses of willing, unwilling, or undecided. These three responses were used as the outcome variable, and multinomial logistic regression analyses were conducted with willingness to be vaccinated as the reference group. Explanatory variables included generalized trust, depression, generalized anxiety, and fear of COVID-19 both at wave 1 and 3, and sociodemographic and health-related variables.

Results: Of the 11,846 valid respondents, 209 (1.8\%) answered that they had already been vaccinated against COVID-19, 7089 (59.8\%) responded that they were willing to be vaccinated, 3498 (29.5\%) responded that they were undecided, and 1053 (8.9\%) responded that they were unwilling to be vaccinated. After adjusting for covariates, we found that: (1) participants with lower levels of generalized trust at wave 1 and 3 were more likely to be undecided or unwilling at wave 3; (2) respondents with moderately severe or severe depression at wave 1 and 3 were more likely to be undecided at wave 3; (3) participants with moderate or severe levels of generalized anxiety at wave 3 but not at wave 1 were more likely to be unwilling at wave 3; and (4) respondents with high levels of fear of COVID-19 at wave 1 and 3 were less likely to be undecided and unwilling at wave 3 .
\end{abstract}

Conclusions: Generalized trust, mental health conditions such as depression and generalized anxiety, and low level of fear of COVID-19 are associated with unwillingness or indecision regarding being vaccinated against COVID-19.

Keywords: COVID-19, Vaccine hesitancy, Generalized trust, Depression, Generalized anxiety, Fear of COVID-19

\section{Background}

COVID-19 has been prevalent around the world since the end of 2019, and many people have lost their lives due to this infectious disease. Many countries have

\footnotetext{
*Correspondence: sekizawa-yoichi@rieti.go.jp

${ }^{1}$ Research Institute of Economy, Trade and Industry, Chiyoda-ku,

Kasumigaseki 1-3-1, Tokyo 100-8901, Japan
}

Full list of author information is available at the end of the article implemented strict measures to control the spread of the disease, such as lockdowns and mandatory wearing of face masks; despite these measures, the disease has spread across the world.

Vaccination is one of the most effective ways of halting the spread of COVID-19. Several vaccines have been developed over a short period of time. Vaccination not only helps prevent individual infections, but 
mass vaccination prevents medical resources from being overwhelmed by lowering the number of critically ill patients and has the potential to halt the pandemic through the achievement of herd immunity. As of November 27, 2021, almost half of the global population has received at least one dose of the COVID19 vaccine, and more than 7 billion doses have been administered worldwide [1].

Vaccine hesitancy has been defined as a "delay in acceptance or refusal of vaccination despite availability of vaccination services" [2]. It creates a serious problem in the promotion of vaccination against COVID-19 [3]. Despite the importance of vaccination, many people are known to have a negative attitude toward it [4]. There is a concern that even if a vaccination program progresses initially, it may stall at some point because of the large number of people in many countries who refuse to be vaccinated [5]. An exploration of the type of people who are less likely to receive a vaccine against COVID-19 is important in order to cover maximum range of people with vaccination. Numerous studies that address the question of which people were reluctant to be vaccinated have been published recently [6-23]. Most of these studies showed that younger people [7, 8, 12, 14-16, 19-23], women $[6,7,9-12,14-16,19-23]$, people with low incomes $[6,11,13-15,18,19,21,22]$, and people with lower levels of education $[6,7,10,11,13-16,18-23]$ were reluctant to receive a vaccine.

Although many predictors of vaccine hesitancy have been identified, some possible predictors remain underexplored. Of these, we examined the associations between vaccination hesitancy and generalized trust, mental health factors such as depression and generalized anxiety, and fear of COVID-19.

Specific trust, such as trust in vaccines, the government, the health system, or professionals, has been sufficiently explored in the context of vaccine hesitancy in general $[24,25]$, and specifically for COVID-19 vaccines (trust in vaccines $[6,16,26-28]$, trust in government [20, $22,26,29,30]$, trust in healthcare system or practitioners [16, 31-33]). By contrast, generalized trust, i.e., a default expectation of goodwill from other people [34] that is often viewed as an important part of social capital [35], has not been sufficiently explored in the context of vaccine hesitancy. We are aware of two studies that focus on the association between generalized trust and COVID-19: Frank and Arim [36] and Ahorsu et al. [31]. Both reported that those who indicated higher levels of generalized trust tended to be more willing to receive the COVID-19 vaccine than those with lower levels of generalized trust. Regarding the association between generalized trust and vaccine hesitancy other than for COVID-19, we found three studies, and all of them concluded that generalized trust was associated with higher willingness to receive a vaccine against influenza [37-39].

Similar to generalized trust, studies that explore the attitudes of people with mental illness toward vaccination against COVID-19 are scarce [40] and inconsistent. To our knowledge, there have been five studies in the United States, China, Germany, and Japan [15, 17, 22, 41, 42]. Three of them found no significant association between depression/anxiety and vaccine hesitancy $[15,17,41]$. By contrast, a study in China that concentrated on people with children found that psychologically distressed subjects were more likely to have vaccine hesitancy related to themselves, their spouses, and their children [42]. Similarly, a study in Japan showed that respondents with severe psychological distress were more likely to have vaccine hesitancy related to the COVID-19 vaccines [22]. More broadly, studies that explore the relationship between depression/anxiety and vaccine hesitancy in general suggest that those who have depression/anxiety tend to be willing to be vaccinated mostly for influenza [43-45].

Regarding fear of COVID-19, several studies have already explored the association between fear of COVID19 and vaccine hesitancy [15, 22, 26, 27, 41, 46-48]. All of them except one [46] found that fear of COVID-19 was positively associated with willingness to be vaccinated against COVID-19. More broadly, studies that explored the relationship between the willingness to receive vaccination and the fear of the disease the vaccine prevents concluded that those who fear the disease tend to be more willing to be vaccinated [49-53]. Nevertheless, a recent model study on the COVID-19 vaccine suggested that the impact of the fear of COVID-19 on the willingness to receive vaccination is small, although it exists [54]. Against this background, it is worthwhile to confirm the robustness of the association between fear of COVID-19 and the willingness to receive vaccination in a country like Japan where the prevalence of COVID-19 cases was lower than in most other countries [1].

In the present study, we explored two things. First, we explored whether generalized trust, mental health factors such as depression and generalized anxiety, and fear of COVID-19 were predictors of COVID-19 vaccine hesitancy. Second, we explored whether the results of the previous studies on predictors, in particular socioeconomic ones, of vaccine hesitancy against COVID-19 can be confirmed with a large data of Japanese people.

\section{Methods}

Research design, participants, and procedures

A longitudinal online survey named "Continuing survey on mental and physical health during the COVID-19 
pandemic" (hereinafter "RIETI questionnaire survey") was planned and organized by the authors as part of a research project of the Research Institute of Economy, Trade and Industry, Japan (RIETI). This longitudinal survey was conducted by NTTCom Online Marketing Solutions Corporation based on the design and instructions of the authors. We used wave 1 and wave 3 of the RIETI questionnaire survey for the present study. Wave 1 was conducted from October 27 to November 6, 2020. Wave 3 was conducted from April 23 to May 6, 2021. Study participants were recruited from the registrants of the corporation or its affiliates. The participants at wave 1 were men and women aged 18-74 years living in Japan. They were extracted so that their demographic composition ratios of sex, age, and distribution of residential prefectures matched the population estimates of the Statistics Bureau of Japan (final estimates, May 2020). Data were excluded for individuals who provided non-existent zip codes, zip codes that did not match their previously registered prefectures, extreme outlying values for height and weight among Japanese adults $(2 \mathrm{~m}$ or taller and weight lower than $35 \mathrm{~kg}$ or in excess of $100 \mathrm{~kg}$, measurements that are outside the national average in Japan), or age differing by 2 years or more from the age previously registered. Respondents who took a very short time (less than $5 \mathrm{~min}$ ) or a very long time ( $10 \mathrm{~h}$ or more) to answer the survey questions were also excluded. The remaining number of individuals after these exclusions was 16,642 (8022 men, 8620 women) and they were established as valid respondents for wave 1 . In the subsequent waves, we asked all valid respondents from wave 1 to respond to the surveys by email. For wave 3 , those who took a very short time (less than $4 \mathrm{~min}$ ) or a very long time ( $10 \mathrm{~h}$ or more) to answer the survey questions were excluded. All communication with study participants, including the questionnaires, was conducted in Japanese.

All individuals who participated in this study provided online informed consent for their participation. The present study was conducted with the approval of the ethics committee of Hiramatsu Memorial Hospital (No: 20200925). All methods were carried out in accordance with relevant guidelines and regulations.

\section{Outcome variables}

The outcome variables used in the present study were based on the following two questions regarding vaccination against COVID-19, which were asked only in wave 3 . The first question asked whether the respondent had been vaccinated. If participants had received at least one dose, they were treated as having been vaccinated. The second question was asked only of those who had not been vaccinated. The question was: "Are you going to receive a vaccine against COVID-19?" It was scored as willing to be vaccinated, unwilling to be vaccinated, or undecided.

\section{Explanatory variables Generalized trust, depression, generalized anxiety, and fear of COVID-19}

To measure generalized trust, we used the Japanese version of the following question from the World Values Survey (WVS) Wave 6 [55]: "Generally speaking, would you say that most people can be trusted or that you need to be very careful in dealing with people?" The available answers were as follows: "Most people can be trusted," "Need to be very careful," and "Don't know." Respondents who selected "Need to be very careful" and "Don't know" were regarded as having low generalized trust. This question has been validated by two experimental studies [56, 57].

Depression was measured using the Patient Health Questionnaire-9 (PHQ-9) [58]. The PHQ-9 is a nineitem self-administered questionnaire designed to assess the presence and severity of depressive symptoms during the past 2 weeks. Example items include "little interest or pleasure in doing things" and "feeling down, depressed, or hopeless." The answers in the PHQ-9 consist of a four-point scale ranging from 0 (not at all) to 3 (nearly every day). The PHQ-9 scores range from 0 to 27 , with higher scores indicating progressively more severe depression $(0-4=$ no depression, $5-9=$ mild depression, $\quad 10-14=$ moderate depression, $15-19=$ moderately severe depression, and $20-27=$ severe depression). Although the original version of the PHQ-9 is in English, a Japanese version was used in the present study [59]. The Japanese version of PHQ-9 has been validated by Muramatsu et al. [60].

Anxiety was measured by means of the Generalized Anxiety Disorder-7 scale (GAD-7) [61]. The GAD-7 is a seven-item, self-administered questionnaire that is designed to measure generalized anxiety symptoms during the past 2 weeks. Example items include "feeling nervous, anxious, or on edge" and "not being able to stop or control worrying." The answers in the GAD-7 consist of a four-point scale ranging from 0 (not at all) to 3 (nearly every day). The GAD-7 scores range from 0 to 21 , with higher scores indicating progressively greater levels of anxiety $(0-4=$ minimal anxiety, $5-9=$ mild anxiety, $10-14=$ moderate anxiety, and $15-21=$ severe anxiety). Although the original version of the GAD-7 is in English, a Japanese version was used in the present study [62]. The Japanese version of GAD-7 has been validated by its translators [62].

The Fear of COVID-19 Scale (FCV-19S) was used to measure fear related to COVID-19 [63]. The FCV-19S is a seven-item, self-reporting questionnaire designed to 
measure the extent to which a person fears COVID-19. Example items included "I am most afraid of coronavirus-19" and "it makes me uncomfortable to think about coronavirus-19." The answers in the FCV-19S consist of a five-point scale ranging from 1 (strongly disagree) to 5 (strongly agree). The FCV-19S scores range from 7 to 35 , with higher scores indicating progressively greater levels of fear related to COVID-19. Since there were no clear categories for the FCV-19S scores, we defined them as follows: $7-15=$ no fear, $16-20=$ mild fear, $21-25=$ moderate fear, and $26-35=$ severe fear. Although the original version of the FCV-19S is in English, a Japanese version was used in the present study [64]. The Japanese version of FCV-19S has been validated by its translators [64].

\section{Other explanatory variables}

Sociodemographic variables included sex, age, level of education, family members living together, employment status, annual household income, bank and saving deposit amount, and place of residence (individual prefectures with a population of 5 million or more [nine prefectures], and eight regions of Japan for those living in other prefectures). Marital status was not included because the question regarding family members living together was similar. For variables relating to health, we used body mass index (BMI) and whether pre-existing conditions (hypertension, dyslipidemia, diabetes, heart disease, renal disease, cancer, respiratory disease, and other) were present. Descriptions of the explanatory variables are shown with the basic statistics in Table 1.

\section{Statistical analysis}

Descriptive statistics were presented with frequency and percentages for categorical variables. Continuous variables were grouped into categorical variables (e.g., age, BMI, household income). Multinomial logistic regression analyses were conducted to estimate the associations between the explanatory variables and the outcome variables. Respondents willing to receive a vaccine at wave 3 (reference group) were compared with (1) those who were undecided regarding vaccination; and (2) those who were unwilling to be vaccinated. We estimated four models. In Model 1 at wave 1 and Model 1 at wave 3, the explanatory variables of primary interest were any one of generalized trust, depression (PHQ-9), generalized anxiety (GAD-7), and fear of COVID-19 (FCV-19S) experienced during wave 1 and wave 3, respectively. In Model 2 at wave 1 and Model 2 at wave 3, the explanatory variables of primary interest were all of generalized trust, PHQ-9, GAD-7, and FCV-19S together, at wave 1 and wave 3, respectively. All other explanatory variables were adjusted for in all models. Generalized trust, PHQ-9, GAD-7, FCV-19S, age, employment status, annual household income, and bank and saving deposit amounts in Models 1 and 2 at wave 1 and wave 3 were those at wave 1 and 3, respectively. Remaining explanatory variables were those at wave 1 . For all models we calculated the relative risk ratio (RRR) and the $95 \%$ confidence interval. Statistical analyses were carried out using STATA 15.0 (STATACorp LLC, Texas, USA).

\section{Results}

\section{Study participants and characteristics}

In wave 3 , we sent surveys via email to the 16,642 valid respondents from wave 1 , and the total number of responses was 13,279 (79.8\%). After excluding those who took a very short time (less than $4 \mathrm{~min}$ ) or a very long time ( $10 \mathrm{~h}$ or more) to answer the survey questions, the number of valid respondents was 11,846 (5965 males and 5881 females), and the mean age was 54.0 years $(S D=14.2$ years). The basic statistics for the outcome variables and all explanatory variables are presented in Table 1.

\section{Attitude toward vaccination}

Of the 11,846 valid respondents, 209 (1.8\%) answered that they had already been vaccinated against COVID$19,7089(59.8 \%)$ responded that they were willing to be vaccinated, 3498 (29.5\%) responded that they were undecided, and 1053 (8.9\%) responded that they were unwilling to be vaccinated (Table 1 ).

\section{Association of generalized trust, depression, generalized anxiety, and fear of COVID-19 with attitudes toward vaccination against COVID-19}

Table 2 presents the results of the adjusted multinomial logistic regression analyses focusing on the association of generalized trust, depression, generalized anxiety, and fear of COVID-19, with attitudes toward vaccination against COVID-19.

Regarding generalized trust, Model 1 at wave 1 and wave 3 both showed that those who answered "Need to be very careful" and "Don't know" were more likely to be undecided, or unwilling to be vaccinated, than those who answered "Most people can be trusted." The results were basically the same in Model 2 at wave 1 and wave 3 , in which depression, generalized anxiety, and fear of COVID-19 were adjusted for.

Regarding depression, Model 1 at wave 1 and wave 3 both showed that those who were mildly depressed, moderately depressed, moderate-severely depressed, and severely depressed were more likely to be undecided than those who were not depressed. These results were basically the same in Model 2 at wave 1 and wave 3, in which generalized trust, generalized anxiety, and fear of COVID-19 were adjusted for, except that mild and 
Table 1 Characteristics and vaccine-related attitudes of study participants

\begin{tabular}{|c|c|c|c|c|c|}
\hline \multirow[t]{2}{*}{ Predictors } & \multirow[t]{2}{*}{ Already vaccinated } & \multicolumn{3}{|c|}{ Not vaccinated } & \multirow[t]{2}{*}{ Total } \\
\hline & & Willing & Undecided & Unwilling & \\
\hline Overall sample & $209(1.8 \%)$ & 7086 (59.8\%) & $3498(29.5 \%)$ & $1053(8.9 \%)$ & 11,846 \\
\hline \multicolumn{6}{|l|}{ Sex } \\
\hline Male & $102(1.7 \%)$ & 3778 (63.3\%) & $1583(26.5 \%)$ & $502(8.4 \%)$ & 5965 \\
\hline Female & $107(1.8 \%)$ & $3308(56.2 \%)$ & 1915 (32.6\%) & $551(9.4 \%)$ & 5881 \\
\hline \multicolumn{6}{|l|}{ Age group, years at wave 1} \\
\hline $65+$ & $28(0.9 \%)$ & 2377 (80.1\%) & $422(14.2 \%)$ & $142(4.8 \%)$ & 2969 \\
\hline $50-64$ & $72(1.7 \%)$ & $2696(61.8 \%)$ & $1262(28.9 \%)$ & $330(7.6 \%)$ & 4360 \\
\hline $30-49$ & $77(2.2 \%)$ & 1615 (46.9\%) & 1347 (39.1\%) & 402 (11.7\%) & 3441 \\
\hline $18-29$ & $32(3.0 \%)$ & $398(37.0 \%)$ & $467(43.4 \%)$ & $179(16.6 \%)$ & 1076 \\
\hline \multicolumn{6}{|l|}{ Age group, years at wave 3} \\
\hline $65+$ & $30(1.0 \%)$ & 2482 (79.8\%) & $451(14.5 \%)$ & $148(4.8 \%)$ & 3111 \\
\hline $50-64$ & $74(1.7 \%)$ & 2671 (60.9\%) & $1296(29.5 \%)$ & 345 (7.9\%) & 4386 \\
\hline $30-49$ & $78(2.3 \%)$ & $1573(46.6 \%)$ & 1330 (39.4\%) & 394 (11.7\%) & 3375 \\
\hline $18-29$ & $27(2.8 \%)$ & $360(37.0 \%)$ & $421(43.2 \%)$ & $166(17.0 \%)$ & 974 \\
\hline \multicolumn{6}{|l|}{ Highest education } \\
\hline Junior/senior high school & $30(0.8 \%)$ & 2171 (57.2\%) & 1215 (32.0\%) & $378(10.0 \%)$ & 3794 \\
\hline Two- or three-year college & $67(2.6 \%)$ & $1446(57.0 \%)$ & $779(30.7 \%)$ & $245(9.7 \%)$ & 2537 \\
\hline Four-year college or higher & $112(2.0 \%)$ & 3469 (62.9\%) & $1504(27.3 \%)$ & $430(7.8 \%)$ & 5515 \\
\hline \multicolumn{6}{|l|}{ Family members living together at wave 1} \\
\hline Living alone & $40(2.0 \%)$ & $1043(52.7 \%)$ & $657(33.2 \%)$ & $241(12.2 \%)$ & 1981 \\
\hline Living only with spouse & $45(1.3 \%)$ & $2479(71.3 \%)$ & $757(21.8 \%)$ & $198(5.7 \%)$ & 3479 \\
\hline Living with children & $79(2.2 \%)$ & $2181(60.8 \%)$ & $1056(29.4 \%)$ & $274(7.6 \%)$ & 3590 \\
\hline Living with parents & $28(1.4 \%)$ & $972(48.3 \%)$ & $756(37.6 \%)$ & $256(12.7 \%)$ & 2012 \\
\hline Three-generation household & $14(2.3 \%)$ & $324(54.4 \%)$ & $205(34.4 \%)$ & $53(8.9 \%)$ & 596 \\
\hline $\begin{array}{l}\text { Other (siblings only, friends, grandparents } \\
\text { and grandchildren etc.) }\end{array}$ & $3(1.6 \%)$ & $87(46.3 \%)$ & $67(35.6 \%)$ & $31(16.5 \%)$ & 188 \\
\hline \multicolumn{6}{|l|}{ Employment at wave 1} \\
\hline Employed & $184(2.6 \%)$ & $4043(57.5 \%)$ & $2163(30.7 \%)$ & $646(9.2 \%)$ & 7036 \\
\hline Homemaker & $11(0.5 \%)$ & $1491(64.8 \%)$ & $659(28.6 \%)$ & $141(6.1 \%)$ & 2302 \\
\hline Not employed (seeking a job) & $3(1.0 \%)$ & $140(47.9 \%)$ & $109(37.3 \%)$ & $40(13.7 \%)$ & 292 \\
\hline Not employed (not seeking a job) & $6(0.3 \%)$ & $1250(68.6 \%)$ & $399(21.9 \%)$ & $166(9.1 \%)$ & 1821 \\
\hline Student & $5(1.9 \%)$ & $98(37.1 \%)$ & $121(45.8 \%)$ & $40(15.2 \%)$ & 264 \\
\hline Other & $0(0.0 \%)$ & $64(48.9 \%)$ & $47(35.9 \%)$ & $20(15.3 \%)$ & 131 \\
\hline \multicolumn{6}{|l|}{ Employment at wave 3} \\
\hline Employed & $185(2.6 \%)$ & $4023(57.1 \%)$ & 2199 (31.2\%) & $639(9.1 \%)$ & 7046 \\
\hline Homemaker & $13(0.6 \%)$ & $1475(65.0 \%)$ & $646(28.5 \%)$ & $134(5.9 \%)$ & 2268 \\
\hline Not employed (seeking a job) & $2(0.6 \%)$ & $159(51.1 \%)$ & $101(32.5 \%)$ & $49(15.8 \%)$ & 311 \\
\hline Not employed (not seeking a job) & $7(0.4 \%)$ & $1278(68.4 \%)$ & $407(21.8 \%)$ & $176(9.4 \%)$ & 1868 \\
\hline Student & $2(0.9 \%)$ & $85(39.4 \%)$ & $97(44.9 \%)$ & $32(14.8 \%)$ & 216 \\
\hline Other & $0(0.0 \%)$ & $66(48.2 \%)$ & $48(35.0 \%)$ & $23(16.8 \%)$ & 137 \\
\hline \multicolumn{6}{|c|}{ Annual household income, million yen at wave 1} \\
\hline$<3$ & $35(1.2 \%)$ & $1611(53.2 \%)$ & $1013(33.4 \%)$ & $372(12.3 \%)$ & 3031 \\
\hline $3-4$ & $42(1.3 \%)$ & $2071(63.6 \%)$ & $872(26.8 \%)$ & $269(8.3 \%)$ & 3254 \\
\hline $5-7$ & $68(2.2 \%)$ & $1887(59.7 \%)$ & $981(31.0 \%)$ & $225(7.1 \%)$ & 3161 \\
\hline$\geq 8$ & $64(2.7 \%)$ & $1517(63.2 \%)$ & $632(26.3 \%)$ & $187(7.8 \%)$ & 2400 \\
\hline \multicolumn{6}{|c|}{ Annual household income, million yen at wave 3} \\
\hline$<3$ & $32(1.0 \%)$ & $1680(54.3 \%)$ & $1008(32.6 \%)$ & $372(12.0 \%)$ & 3092 \\
\hline $3-4$ & $45(1.4 \%)$ & $2024(62.8 \%)$ & $888(27.5 \%)$ & $267(8.3 \%)$ & 3224 \\
\hline
\end{tabular}


Table 1 (continued)

\begin{tabular}{|c|c|c|c|c|c|}
\hline \multirow[t]{2}{*}{ Predictors } & \multirow[t]{2}{*}{ Already vaccinated } & \multicolumn{3}{|c|}{ Not vaccinated } & \multirow[t]{2}{*}{ Tota } \\
\hline & & Willing & Undecided & Unwilling & \\
\hline $5-7$ & $67(2.1 \%)$ & $1885(59.6 \%)$ & $988(31.2 \%)$ & $224(7.1 \%)$ & 3164 \\
\hline$\geq 8$ & $65(2.7 \%)$ & $1497(63.3 \%)$ & $614(26.0 \%)$ & $190(8.0 \%)$ & 2366 \\
\hline \multicolumn{6}{|c|}{ Bank and savings deposit amount, million yen at wave 1} \\
\hline$<1$ & $63(2.0 \%)$ & $1529(49.5 \%)$ & $1121(36.3 \%)$ & $373(12.1 \%)$ & 3086 \\
\hline $1-3$ & $39(1.7 \%)$ & $1286(56.5 \%)$ & $743(32.6 \%)$ & $209(9.2 \%)$ & 2277 \\
\hline $4-9$ & $47(2.0 \%)$ & $1421(60.0 \%)$ & $706(29.8 \%)$ & $196(8.3 \%)$ & 2370 \\
\hline$\geq 10$ & $60(1.5 \%)$ & $2850(69.3 \%)$ & $928(22.6 \%)$ & $275(6.7 \%)$ & 4113 \\
\hline \multicolumn{6}{|c|}{ Bank and savings deposit amount, million yen at wave 3} \\
\hline$<1$ & $51(1.6 \%)$ & $1538(49.1 \%)$ & $1153(36.8 \%)$ & $390(12.5 \%)$ & 3132 \\
\hline $1-3$ & $51(2.3 \%)$ & $1258(56.8 \%)$ & $714(32.2 \%)$ & $191(8.6 \%)$ & 2214 \\
\hline $4-9$ & $39(1.7 \%)$ & $1417(61.1 \%)$ & $687(29.6 \%)$ & $176(7.6 \%)$ & 2319 \\
\hline$\geq 10$ & $68(1.6 \%)$ & $2873(68.7 \%)$ & $944(22.6 \%)$ & $296(7.1 \%)$ & 4181 \\
\hline \multicolumn{6}{|l|}{ BMl at wave 1} \\
\hline$<18.5$ & $23(1.6 \%)$ & $739(50.4 \%)$ & $524(35.7 \%)$ & $181(12.3 \%)$ & 1467 \\
\hline $18.5-24.9$ & $148(1.8 \%)$ & $4930(60.5 \%)$ & $2377(29.2 \%)$ & $693(8.5 \%)$ & 8148 \\
\hline $25.0-29.9$ & $32(1.7 \%)$ & $1210(64.3 \%)$ & $487(25.9 \%)$ & $153(8.1 \%)$ & 1882 \\
\hline$\geq 30.0$ & $6(1.7 \%)$ & $207(59.3 \%)$ & $110(31.5 \%)$ & $26(7.4 \%)$ & 349 \\
\hline \multicolumn{6}{|l|}{ Pre-existing condition at wave 1} \\
\hline Hypertension & $34(1.6 \%)$ & $1577(74.8 \%)$ & 402 (19.1\%) & $96(4.6 \%)$ & 2109 \\
\hline Dyslipidemia & $25(2.1 \%)$ & $910(76.2 \%)$ & $200(16.8 \%)$ & $59(4.9 \%)$ & 1194 \\
\hline Diabetes & $9(1.4 \%)$ & $482(72.5 \%)$ & $136(20.5 \%)$ & $38(5.7 \%)$ & 665 \\
\hline Heart disease & $7(2.2 \%)$ & $234(74.3 \%)$ & $56(17.8 \%)$ & $18(5.7 \%)$ & 315 \\
\hline Renal disease & $2(2.1 \%)$ & $66(68.8 \%)$ & $23(24.0 \%)$ & $5(5.2 \%)$ & 96 \\
\hline Cancer & $4(2.0 \%)$ & $146(73.7 \%)$ & $36(18.2 \%)$ & $12(6.1 \%)$ & 198 \\
\hline Respiratory disease & $5(1.8 \%)$ & $180(65.2 \%)$ & $66(23.9 \%)$ & $25(9.1 \%)$ & 276 \\
\hline Other & $2(1.2 \%)$ & $102(60.0 \%)$ & $50(29.4 \%)$ & $16(9.4 \%)$ & 170 \\
\hline \multicolumn{6}{|l|}{ Generalized trust at wave 1} \\
\hline Most people can be trusted & $70(1.8 \%)$ & $2604(67.9 \%)$ & $929(24.2 \%)$ & $234(6.1 \%)$ & 3837 \\
\hline Need to be very careful & $132(1.8 \%)$ & $4220(56.4 \%)$ & $2369(31.7 \%)$ & $760(10.2 \%)$ & 7481 \\
\hline Don't know & $7(1.3 \%)$ & $262(49.6 \%)$ & $200(37.9 \%)$ & $59(11.2 \%)$ & 528 \\
\hline \multicolumn{6}{|l|}{ Generalized trust at wave 3} \\
\hline Most people can be trusted & $63(1.7 \%)$ & $2532(69.5 \%)$ & $822(22.6 \%)$ & $225(6.2 \%)$ & 3642 \\
\hline Need to be very careful & $139(1.8 \%)$ & $4287(55.9 \%)$ & $2473(32.3 \%)$ & $765(10.0 \%)$ & 7664 \\
\hline Don't know & $7(1.3 \%)$ & $267(49.4 \%)$ & $203(37.6 \%)$ & $63(11.7 \%)$ & 540 \\
\hline \multicolumn{6}{|l|}{ Depression (PHQ-9) at wave 1} \\
\hline None $(0-4)$ & $113(1.5 \%)$ & $4761(65.1 \%)$ & $1899(26.0 \%)$ & $540(7.4 \%)$ & 7313 \\
\hline Mild (5-9) & $56(2.0 \%)$ & $1533(54.2 \%)$ & $949(33.6 \%)$ & $290(10.3 \%)$ & 2828 \\
\hline Moderate (10-14) & $24(2.4 \%)$ & $497(49.2 \%)$ & $375(37.1 \%)$ & $115(11.4 \%)$ & 1011 \\
\hline Moderately severe (15-19) & $12(2.5 \%)$ & $209(44.0 \%)$ & $183(38.5 \%)$ & 71 (14.9\%) & 475 \\
\hline Severe (20-27) & $4(1.8 \%)$ & $86(39.3 \%)$ & $92(42.0 \%)$ & $37(16.9 \%)$ & 219 \\
\hline \multicolumn{6}{|l|}{ Depression (PHQ-9) at wave 3} \\
\hline None $(0-4)$ & $110(1.5 \%)$ & $4784(65.0 \%)$ & $1918(26.1 \%)$ & $545(7.4 \%)$ & 7357 \\
\hline Mild (5-9) & $51(1.9 \%)$ & $1511(55.2 \%)$ & $919(33.6 \%)$ & $257(9.4 \%)$ & 2738 \\
\hline Moderate (10-14) & $29(2.9 \%)$ & $487(48.7 \%)$ & $352(35.2 \%)$ & $131(13.1 \%)$ & 999 \\
\hline Moderately severe (15-19) & $10(2.1 \%)$ & $209(43.1 \%)$ & $192(39.6 \%)$ & $74(15.3 \%)$ & 485 \\
\hline Severe $(20-27)$ & $9(3.4 \%)$ & $95(35.6 \%)$ & $117(43.8 \%)$ & $46(17.2 \%)$ & 267 \\
\hline \multicolumn{6}{|c|}{ Generalized anxiety (GAD-7) at wave 1} \\
\hline Minimal (0-4) & $140(1.6 \%)$ & 5628 (63.0\%) & $2453(27.5 \%)$ & 714 (8.0\%) & 8935 \\
\hline
\end{tabular}


Table 1 (continued)

\begin{tabular}{|c|c|c|c|c|c|}
\hline \multirow[t]{2}{*}{ Predictors } & \multirow[t]{2}{*}{ Already vaccinated } & \multicolumn{3}{|c|}{ Not vaccinated } & \multirow[t]{2}{*}{ Total } \\
\hline & & Willing & Undecided & Unwilling & \\
\hline Mild (5-9) & $38(2.0 \%)$ & $998(52.8 \%)$ & $662(35.0 \%)$ & $192(10.2 \%)$ & 1890 \\
\hline Moderate (10-14) & $23(3.3 \%)$ & $321(46.0 \%)$ & $260(37.2 \%)$ & $94(13.5 \%)$ & 698 \\
\hline Severe $(15-21)$ & $8(2.5 \%)$ & $139(43.0 \%)$ & $123(38.1 \%)$ & $53(16.4 \%)$ & 323 \\
\hline \multicolumn{6}{|c|}{ Generalized anxiety (GAD-7) at wave 3} \\
\hline Minimal (0-4) & $133(1.5 \%)$ & $5588(63.3 \%)$ & $2412(27.3 \%)$ & $690(7.8 \%)$ & 8823 \\
\hline Mild (5-9) & $43(2.2 \%)$ & $1032(52.5 \%)$ & $694(35.3 \%)$ & $197(10.0 \%)$ & 1966 \\
\hline Moderate (10-14) & $22(3.4 \%)$ & $306(47.1 \%)$ & $226(34.8 \%)$ & $96(14.8 \%)$ & 650 \\
\hline Severe (15-21) & $11(2.7 \%)$ & $160(39.3 \%)$ & $166(40.8 \%)$ & $70(17.2 \%)$ & 407 \\
\hline \multicolumn{6}{|c|}{ Fear of COVID-19 (FCV-19S) at wave 1} \\
\hline No fear (7-15) & $65(1.8 \%)$ & $2066(56.7 \%)$ & $1056(29.0 \%)$ & $456(12.5 \%)$ & 3643 \\
\hline Mild (16-20) & $79(1.9 \%)$ & $2556(62.8 \%)$ & $1152(28.3 \%)$ & $285(7.0 \%)$ & 4072 \\
\hline Moderate (21-25) & $45(1.5 \%)$ & $1761(59.5 \%)$ & $934(31.6 \%)$ & $218(7.4 \%)$ & 2958 \\
\hline Severe $(26-35)$ & $20(1.7 \%)$ & 703 (59.9\%) & $356(30.3 \%)$ & $94(8.0 \%)$ & 1173 \\
\hline \multicolumn{6}{|c|}{ Fear of COVID-19 (FCV-19S) At wave 3} \\
\hline No fear (7-15) & $55(1.8 \%)$ & $1686(54.7 \%)$ & $869(28.2 \%)$ & $472(15.3 \%)$ & 3082 \\
\hline Mild (16-20) & $72(1.8 \%)$ & $2514(62.7 \%)$ & 1159 (28.9\%) & $262(6.5 \%)$ & 4007 \\
\hline Moderate (21-25) & $56(1.7 \%)$ & $1992(60.1 \%)$ & $1041(31.4 \%)$ & $228(6.9 \%)$ & 3317 \\
\hline Severe (26-35) & $26(1.8 \%)$ & $894(62.1 \%)$ & $429(29.8 \%)$ & $91(6.3 \%)$ & 1440 \\
\hline \multicolumn{6}{|c|}{ Region of residence at wave 1} \\
\hline Tokyo & $21(1.7 \%)$ & $718(57.9 \%)$ & $368(29.7 \%)$ & $133(10.7 \%)$ & 1240 \\
\hline Kanagawa & $9(1.0 \%)$ & $528(61.5 \%)$ & $266(31.0 \%)$ & $56(6.5 \%)$ & 859 \\
\hline Osaka & $10(1.2 \%)$ & $520(61.3 \%)$ & $240(28.3 \%)$ & $78(9.2 \%)$ & 848 \\
\hline Aichi & $10(1.4 \%)$ & $449(61.8 \%)$ & $203(28.0 \%)$ & $64(8.8 \%)$ & 726 \\
\hline Saitama & $9(1.3 \%)$ & $413(59.8 \%)$ & $220(31.8 \%)$ & $49(7.1 \%)$ & 691 \\
\hline Chiba & $8(1.3 \%)$ & 374 (60.9\%) & 165 (26.9\%) & 67 (10.9\%) & 614 \\
\hline Hyogo & $3(0.5 \%)$ & $341(62.1 \%)$ & $153(27.9 \%)$ & $52(9.5 \%)$ & 549 \\
\hline Hokkaido & $7(1.3 \%)$ & $328(61.9 \%)$ & $146(27.5 \%)$ & $49(9.2 \%)$ & 530 \\
\hline Fukuoka & $5(1.1 \%)$ & $259(59.3 \%)$ & $120(27.5 \%)$ & $53(12.1 \%)$ & 437 \\
\hline Tohoku region & $14(1.7 \%)$ & 509 (63.1\%) & $220(27.3 \%)$ & 64 (7.9\%) & 807 \\
\hline North Kanto & $14(2.3 \%)$ & $341(56.7 \%)$ & 195 (32.4\%) & $51(8.5 \%)$ & 601 \\
\hline Hokuriku & $9(1.9 \%)$ & $280(60.6 \%)$ & $138(29.9 \%)$ & $35(7.6 \%)$ & 462 \\
\hline Chubu & $16(1.9 \%)$ & $492(59.3 \%)$ & $260(31.4 \%)$ & $61(7.4 \%)$ & 829 \\
\hline Kinki & $15(1.8 \%)$ & $513(60.1 \%)$ & $240(28.1 \%)$ & 85 (10.0\%) & 853 \\
\hline Chugoku & $19(2.8 \%)$ & $384(55.8 \%)$ & $230(33.4 \%)$ & 55 (8.0\%) & 688 \\
\hline Shikoku & $12(3.5 \%)$ & $214(62.4 \%)$ & $87(25.4 \%)$ & $30(8.7 \%)$ & 343 \\
\hline Kyushu and Okinawa & $28(3.6 \%)$ & $423(55.0 \%)$ & $247(32.1 \%)$ & $71(9.2 \%)$ & 769 \\
\hline
\end{tabular}

moderate depression lost significance in Model 2 at wave 3 . With regard to unwillingness, Model 1 at wave 1 and wave 3 showed that those who were mildly depressed (at wave 1 only), moderately depressed (at wave 3 only), moderate-severely depressed, and severely depressed were more likely to be unwilling to be vaccinated than those who were not depressed. In Model 2 at wave 1 and wave 3 , in which generalized trust, generalized anxiety, and fear of COVID-19 were adjusted for, there were no significant associations between depression and unwillingness to be vaccinated except that mild depression at wave 1 and moderate depression at wave 3 remained significant.

Regarding generalized anxiety, Model 1 at wave 1 and wave 3 showed that those who were mildly anxious and severely anxious (at wave 3 only) were more likely to be undecided about whether to receive a vaccine than respondents who had minimal levels of anxiety. In Model 2 at wave 1 and wave 3, in which generalized trust, depression, and fear of COVID-19 were adjusted 
$\bar{\delta} \bar{\delta}$

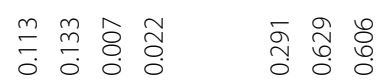

$\begin{array}{lll}0 & 0 \\ 0 & 0 \\ 0 & 0 \\ 0 & 0 & v\end{array}$

$\begin{array}{ll}\overline{8} & \overline{8} \\ \dot{0} & 0 \\ v & v\end{array}$

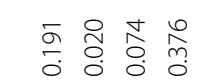

\begin{tabular}{l}
$\bar{\sigma} \sigma$ \\
$\overline{1}$ \\
\multirow{1}{*}{} \\
$m$ \\
$=$
\end{tabular}

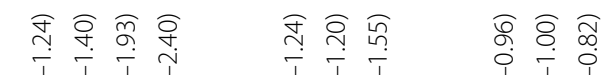

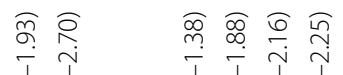

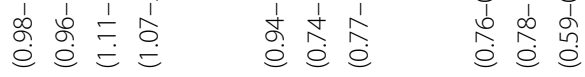

$\stackrel{\infty}{m} \stackrel{\frac{1}{\sigma}}{=}$

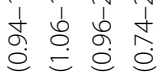

蒿

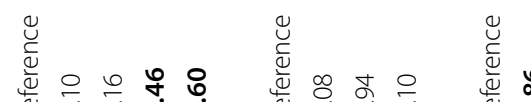

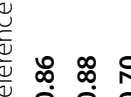

岑

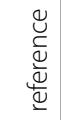

$\begin{array}{ll}\bar{\sigma} & \overline{8} \\ \dot{0} & 0 \\ \vee & \vee\end{array}$

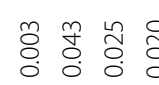

先

$\begin{array}{lll}0 & \\ 0 & 0 & 0 \\ 0 & 0 & 0 \\ 0 & 0\end{array}$

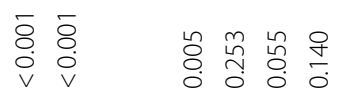

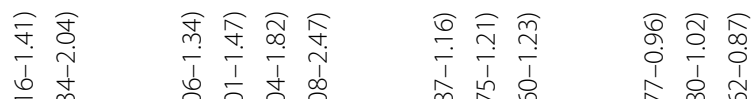

$\stackrel{+}{\stackrel{1}{=}} \stackrel{m}{=}$

足

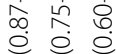

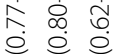

๙্.

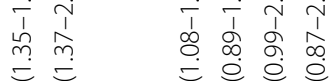

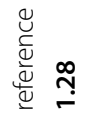

岂

œ

岑

$\overline{8}$
$\dot{0}$
$\mathrm{v}$
$\mathrm{g}$

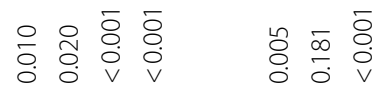

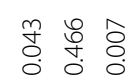

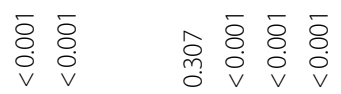

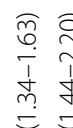

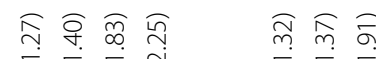

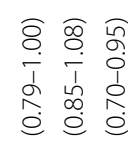

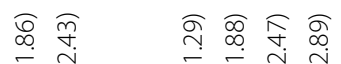

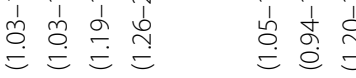

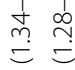

مै ণิ

亗

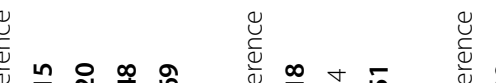

选

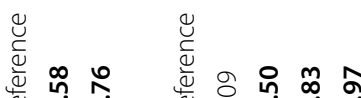

$\bar{\delta}$
$\dot{\delta}$
$\dot{0}$
$v$

$\begin{array}{llll}\text { v } & 0 & 0 & 0 \\ 0 & 0 & 0 \\ 0 & 0\end{array}$

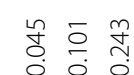

$\begin{array}{lll}\circ & 0 \\ 0 & 0 & 0 \\ 0 & 0 & 0 \\ 0 & 0 & 0\end{array}$

$\begin{array}{ll}\overline{8} & \overline{8} \\ 0 & 0 \\ \dot{0} & v\end{array}$

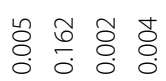

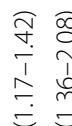

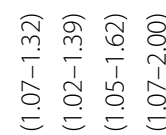

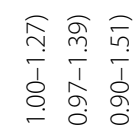

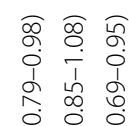

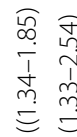

og

峁

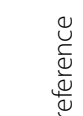

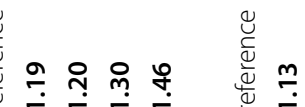

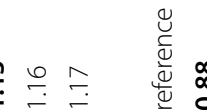
$\begin{array}{cccc}\infty & & \\ \infty & 0 & 0 \\ 0 & 0 & 0 & 0 \\ 0 & 0 & 0\end{array}$

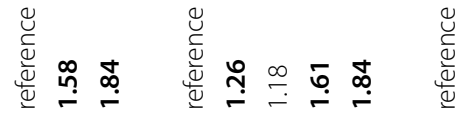

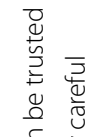

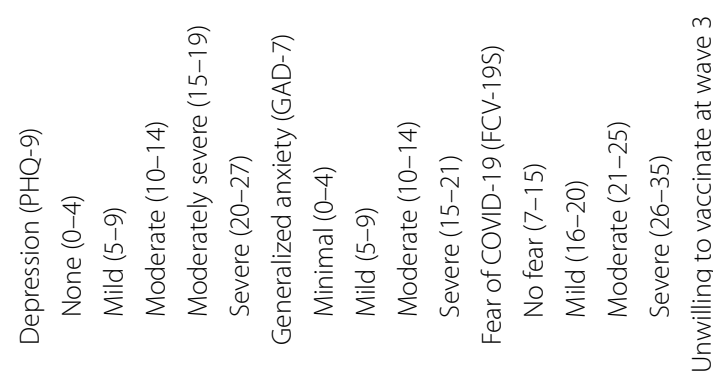

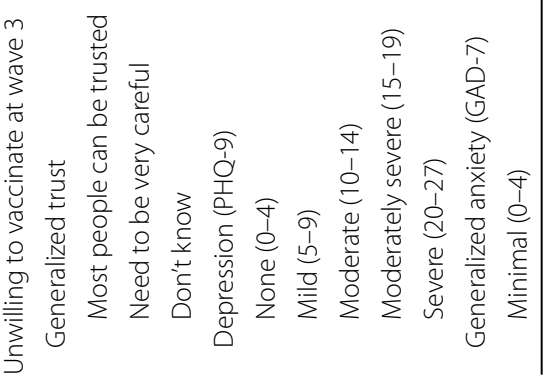




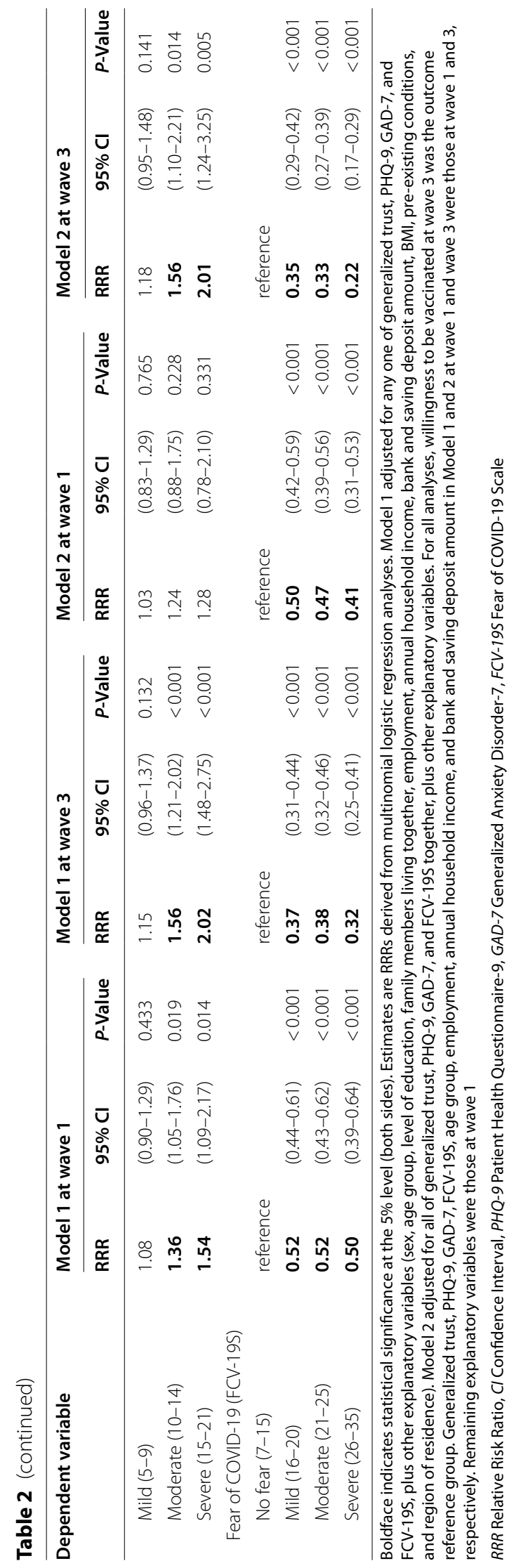


for, there were no significant associations between generalized anxiety and indecisiveness regarding vaccination. With regard to unwillingness, Model 1 at wave 1 and wave 3 showed that those who were moderately anxious and severely anxious were more likely to be unwilling to be vaccinated than those who had a minimal level of anxiety. In Model 2 at wave 1 and wave 3, in which generalized trust, depression, and fear of COVID-19 were adjusted for, moderate anxiety and severe anxiety at wave 1 lost significance, but those at wave 3 remained significant.

Regarding fear of COVID-19, Model 1 at wave 1 and wave 3 showed that participants with mild and severe fear of COVID-19 were less likely to be undecided regarding vaccination than those who had no fear of COVID-19. In Model 2 at wave 1 and wave 3, in which generalized trust, depression, and generalized anxiety were adjusted for, the results were basically the same except that moderate fear of COVID-19 at wave 3 gained significance. With regard to unwillingness, Model 1 at wave 1 and at wave 3 showed that respondents with mild, moderate, and severe fear of COVID-19 were less likely to be unwilling to be vaccinated than those who had no fear of COVID-19. In Model 2 at wave 1 and wave 3 , in which generalized trust, depression, and fear of COVID-19 were adjusted for, the results remained basically the same.

\section{Association of other explanatory variables with attitudes toward vaccination against COVID-19}

The results of Model 2 at wave 1, in which all explanatory variables were included, are shown in Table 3. Being female (undecided only), having a lower level of education, low household income, low savings, and low BMI were positively associated with indecisiveness or unwillingness. Older age, living only with a spouse, and having hypertension or dyslipidemia were negatively associated with indecisiveness and unwillingness. The results of Model 2 at wave 3 were basically the same (shown in Supplementary Table 1).

\section{Discussion}

In the present study, we explored whether generalized trust, mental health factors such as depression and generalized anxiety, and fear of COVID-19 are predictors of vaccine hesitancy against COVID-19. We used the data on wave 1 (between October 27 and November 6, 2020) and wave 3 (between April 23 and May 6, 2021) of a longitudinal online survey carried out in Japan. We found that: (1) respondents who had lower levels of generalized trust at wave 1 and 3 were more likely to be undecided or unwilling to be vaccinated at wave 3 ; (2) respondents with moderately severe or severe levels of depression at wave 1 and 3 were more likely to be undecided about being vaccinated at wave 3 ; (3) respondents with moderate or severe levels of generalized anxiety at wave 3 but not at wave 1 were more likely to be unwilling to be vaccinated at wave 3; and (4) participants with high levels of fear of COVID-19 at wave 1 and 3 were less likely to be undecided or unwilling to be vaccinated at wave 3 . In addition to these results, our findings confirmed the results of previous studies that found that younger people, women, people with low incomes, and people with lower levels of education were more likely to be undecided or unwilling to be vaccinated.

The wave 3 survey, in which the question on vaccine hesitancy was asked, was conducted between April 23 and May 6, 2021, in Japan, where vaccination against COVID-19 for medical personnel began on February 17, 2021, a few months later than Europe and the United States. On April 12, vaccination for persons aged 65 and over was initiated. As of April 23, the shares of the Japanese populations that had received at least one dose of the COVID-19 vaccine and been fully vaccinated against COVID-19 were 1.7 and $0.7 \%$, respectively [1]. As of April 23, total cases of COVID-19 per million people were 4426 and total deaths per million people were 78 in Japan [1]. Hence, the ratios of those who were vaccinated against COVID-19 and casualties due to COVID-19 were lower in Japan than in many other countries. The declaration of a state of emergency was in effect in several areas of Japan including Tokyo and Osaka at that time in order to inhibit the spread of COVID-19. The results of the present study must be interpreted against this background.

Regarding generalized trust in people, the present study showed that participants with low levels of generalized trust at waves 1 and 3 were more likely to be undecided or unwilling to be vaccinated at wave 3 . Wave 1 was approximately 6 months before wave 3 . Therefore, the present study suggest that the level of generalized trust predicts vaccine hesitancy 6 months later. This finding is consistent with the two previous studies that reported that those who indicated a higher level of generalized trust tended to be more willing to get a COVID-19 vaccine than those with a lower level of generalized trust [31, 36]. The relationship between generalized trust and vaccine hesitancy in general was addressed in a systematic review by Larson et al. [25]. However, only three of the reviewed studies examined the relationship between generalized trust and vaccination, and one of them examined trust in the government. Two studies [37, 39] referred to in Larson et al. and another study [38] reported findings consistent with the present study; that is, participants with high generalized trust were more willing to be vaccinated against influenza. Hence, studies thus far, including the present one, have consistently shown that generalized trust is positively associated with vaccine acceptance. 
Table 3 Results of the fully adjusted multinomial logistic regression analyses at wave 1

\begin{tabular}{|c|c|c|c|c|c|c|}
\hline \multirow[t]{2}{*}{ Predictors } & \multicolumn{3}{|c|}{ Undecided } & \multicolumn{3}{|l|}{ Unwilling } \\
\hline & RRR & $95 \% \mathrm{Cl}$ & $P$-value & RRR & $95 \% \mathrm{Cl}$ & $P$-value \\
\hline \multicolumn{7}{|l|}{ Sex } \\
\hline Male & reference & & & reference & & \\
\hline Female & 1.19 & $(1.07-1.32)$ & 0.001 & 1.13 & $(0.96-1.32)$ & 0.145 \\
\hline \multicolumn{7}{|l|}{ Age group, years } \\
\hline $65+$ & reference & & & reference & & \\
\hline $50-64$ & 2.35 & $(2.04-2.70)$ & $<0.001$ & 1.82 & $(1.44-2.29)$ & $<0.001$ \\
\hline $30-49$ & 3.58 & $(3.06-4.19)$ & $<0.001$ & 3.04 & $(2.35-3.91)$ & $<0.001$ \\
\hline $18-29$ & 4.16 & $(3.35-5.16)$ & $<0.001$ & 4.46 & $(3.23-6.15)$ & $<0.001$ \\
\hline \multicolumn{7}{|l|}{ Highest education } \\
\hline Junior/senior high school & 1.21 & $(1.09-1.35)$ & $<0.001$ & 1.32 & $(1.11-1.56)$ & 0.001 \\
\hline Two- or three-year college & 1.08 & $(0.96-1.22)$ & 0.185 & 1.28 & $(1.06-1.54)$ & 0.009 \\
\hline Four-year college or higher & reference & & & reference & & \\
\hline \multicolumn{7}{|l|}{ Family members living together } \\
\hline Living alone & 1.33 & $(1.15-1.54)$ & $<0.001$ & 1.46 & $(1.16-1.84)$ & 0.001 \\
\hline Living only with spouses & reference & & & reference & & \\
\hline Living with children & 1.15 & $(1.02-1.29)$ & 0.027 & 1.20 & $(0.98-1.47)$ & 0.082 \\
\hline Living with parents & 1.34 & $(1.16-1.55)$ & $<0.001$ & 1.46 & $(1.16-1.84)$ & 0.001 \\
\hline Three generation household & 1.49 & $(1.21-1.83)$ & $<0.001$ & 1.40 & $(0.99-1.98)$ & 0.059 \\
\hline $\begin{array}{l}\text { Others (siblings only, friends, grandpar- } \\
\text { ents and grandchildren etc.) }\end{array}$ & 1.38 & $(0.97-1.96)$ & 0.077 & 1.88 & $(1.17-3.00)$ & 0.009 \\
\hline \multicolumn{7}{|l|}{ Employment } \\
\hline Employed & reference & & & reference & & \\
\hline Homemaker & 1.09 & $(0.95-1.25)$ & 0.208 & 0.86 & $(0.68-1.08)$ & 0.198 \\
\hline Not employed (seeking a job) & 0.98 & $(0.74-1.28)$ & 0.858 & 0.98 & $(0.67-1.45)$ & 0.937 \\
\hline Not employed (not seeking job) & 1.06 & $(0.91-1.24)$ & 0.437 & 1.29 & $(1.03-1.61)$ & 0.026 \\
\hline Student & 0.88 & $(0.64-1.21)$ & 0.420 & 0.72 & $(0.46-1.11)$ & 0.137 \\
\hline Other & 1.60 & $(1.05-2.42)$ & 0.028 & 1.99 & $(1.14-3.47)$ & 0.015 \\
\hline \multicolumn{7}{|l|}{ Annual household income, million yen } \\
\hline$<3$ & 1.35 & $(1.15-1.58)$ & $<0.001$ & 1.39 & $(1.09-1.77)$ & 0.008 \\
\hline $3-4$ & 0.99 & $(0.86-1.14)$ & 0.871 & 0.96 & $(0.77-1.20)$ & 0.730 \\
\hline $5-7$ & 1.12 & $(0.98-1.28)$ & 0.086 & 0.87 & $(0.70-1.08)$ & 0.216 \\
\hline$\geq 8$ & reference & & & reference & & \\
\hline \multicolumn{7}{|c|}{ Bank and saving deposit amount, million yen } \\
\hline$<1$ & 1.44 & $(1.27-1.64)$ & $<0.001$ & 1.45 & $(1.18-1.77)$ & $<0.001$ \\
\hline $1-3$ & 1.23 & $(1.08-1.40)$ & 0.002 & 1.13 & $(0.92-1.40)$ & 0.242 \\
\hline $4-9$ & 1.23 & $(1.08-1.39)$ & 0.001 & 1.13 & $(0.92-1.39)$ & 0.238 \\
\hline$\geq 10$ & reference & & & reference & & \\
\hline \multicolumn{7}{|l|}{ BMl } \\
\hline$<18.5$ & 1.17 & $(1.03-1.34)$ & 0.019 & 1.34 & $(1.10-1.62)$ & 0.003 \\
\hline $18.5-24.9$ & reference & & & reference & & \\
\hline $25.0-29.9$ & 0.94 & $(0.83-1.07)$ & 0.341 & 1.02 & $(0.84-1.25)$ & 0.827 \\
\hline$\geq 30.0$ & 0.97 & $(0.75-1.26)$ & 0.833 & 0.76 & $(0.49-1.19)$ & 0.232 \\
\hline \multicolumn{7}{|l|}{ Pre-existing condition } \\
\hline Hypertension & 0.79 & $(0.69-0.90)$ & $<0.001$ & 0.62 & $(0.49-0.78)$ & $<0.001$ \\
\hline Dyslipidemia & 0.62 & $(0.52-0.73)$ & $<0.001$ & 0.68 & $(0.51-0.91)$ & 0.009 \\
\hline Diabetes & 0.95 & $(0.77-1.17)$ & 0.610 & 0.88 & $(0.62-1.27)$ & 0.501 \\
\hline Heart disease & 0.86 & $(0.63-1.17)$ & 0.338 & 0.94 & $(0.56-1.56)$ & 0.802 \\
\hline Renal disease & 1.12 & $(0.67-1.85)$ & 0.674 & 0.70 & $(0.27-1.81)$ & 0.467 \\
\hline
\end{tabular}


Table 3 (continued)

\begin{tabular}{|c|c|c|c|c|c|c|}
\hline \multirow[t]{2}{*}{ Predictors } & \multicolumn{3}{|c|}{ Undecided } & \multicolumn{3}{|l|}{ Unwilling } \\
\hline & RRR & $95 \% \mathrm{Cl}$ & $P$-value & RRR & $95 \% \mathrm{Cl}$ & $P$-value \\
\hline Cancer & 0.74 & $(0.50-1.09)$ & 0.126 & 0.82 & $(0.44-1.52)$ & 0.522 \\
\hline Respiratory disease & 0.97 & $(0.71-1.31)$ & 0.838 & 1.23 & $(0.78-1.92)$ & 0.369 \\
\hline Other condition & 1.03 & $(0.71-1.47)$ & 0.890 & 1.02 & $(0.58-1.77)$ & 0.952 \\
\hline \multicolumn{7}{|l|}{ Region of residence } \\
\hline Tokyo & reference & & & reference & & \\
\hline Kanagawa & 1.05 & $(0.85-1.29)$ & 0.652 & 0.64 & $(0.45-0.90)$ & 0.011 \\
\hline Osaka & 0.93 & $(0.75-1.14)$ & 0.479 & 0.83 & $(0.60-1.13)$ & 0.237 \\
\hline Aichi & 0.90 & $(0.72-1.12)$ & 0.343 & 0.80 & $(0.57-1.11)$ & 0.180 \\
\hline Saitama & 1.12 & $(0.90-1.39)$ & 0.319 & 0.69 & $(0.48-1.00)$ & 0.049 \\
\hline Chiba & 0.93 & $(0.73-1.17)$ & 0.525 & 1.05 & $(0.75-1.47)$ & 0.776 \\
\hline Hyogo & 0.92 & $(0.72-1.17)$ & 0.496 & 0.87 & $(0.61-1.25)$ & 0.457 \\
\hline Hokkaido & 0.86 & $(0.67-1.10)$ & 0.225 & 0.80 & $(0.55-1.15)$ & 0.227 \\
\hline Fukuoka & 0.84 & $(0.64-1.09)$ & 0.187 & 1.01 & $(0.70-1.46)$ & 0.964 \\
\hline Tohoku region & 0.74 & $(0.59-0.91)$ & 0.005 & 0.61 & $(0.44-0.86)$ & 0.004 \\
\hline North Kanto & 1.15 & $(0.92-1.45)$ & 0.228 & 0.84 & $(0.59-1.22)$ & 0.365 \\
\hline Hokuriku & 0.92 & $(0.71-1.18)$ & 0.501 & 0.64 & $(0.42-0.97)$ & 0.037 \\
\hline Chubu & 1.06 & $(0.86-1.30)$ & 0.593 & 0.67 & $(0.47-0.93)$ & 0.019 \\
\hline Kinki & 0.96 & $(0.78-1.19)$ & 0.713 & 0.96 & $(0.71-1.31)$ & 0.817 \\
\hline Chugoku & 1.30 & $(1.05-1.63)$ & 0.018 & 0.89 & $(0.63-1.27)$ & 0.535 \\
\hline Shikoku & 0.81 & $(0.60-1.09)$ & 0.156 & 0.81 & $(0.52-1.27)$ & 0.358 \\
\hline Kyushu and Okinawa & 1.10 & $(0.89-1.36)$ & 0.389 & 0.86 & $(0.61-1.19)$ & 0.354 \\
\hline \multicolumn{7}{|l|}{ Generalized trust } \\
\hline Most people can be trusted & reference & & & reference & & \\
\hline Need to be very careful & 1.28 & $(1.16-1.41)$ & $<0.001$ & 1.59 & $(1.35-1.87)$ & $<0.001$ \\
\hline Don't know & 1.65 & $(1.34-2.04)$ & $<0.001$ & 1.90 & $(1.37-2.63)$ & $<0.001$ \\
\hline \multicolumn{7}{|l|}{ Depression (PHQ-9) } \\
\hline None (0-4) & reference & & & reference & & \\
\hline Mild (5-9) & 1.19 & $(1.06-1.34)$ & 0.003 & 1.30 & $(1.08-1.55)$ & 0.005 \\
\hline Moderate (10-14) & 1.22 & $(1.01-1.47)$ & 0.043 & 1.19 & $(0.89-1.59)$ & 0.253 \\
\hline Moderately severe (15--19) & 1.38 & $(1.04-1.82)$ & 0.025 & 1.48 & $(0.99-2.22)$ & 0.055 \\
\hline Severe $(20-27)$ & 1.63 & $(1.08-2.47)$ & 0.020 & 1.53 & $(0.87-2.71)$ & 0.140 \\
\hline \multicolumn{7}{|l|}{ Generalized anxiety (GAD-7) } \\
\hline Minimal (0-4) & reference & & & reference & & \\
\hline Mild (5-9) & 1.00 & $(0.87-1.16)$ & 0.965 & 1.03 & $(0.83-1.29)$ & 0.765 \\
\hline Moderate (10-14) & 0.96 & $(0.75-1.21)$ & 0.706 & 1.24 & $(0.88-1.75)$ & 0.228 \\
\hline Severe (15-21) & 0.86 & $(0.60-1.23)$ & 0.402 & 1.28 & $(0.78-2.10)$ & 0.331 \\
\hline \multicolumn{7}{|l|}{ Fear of COVID-19 (FCV-19S) } \\
\hline No fear (7-15) & reference & & & reference & & \\
\hline Mild (16-20) & 0.86 & $(0.77-0.96)$ & 0.006 & 0.50 & $(0.42-0.59)$ & $<0.001$ \\
\hline Moderate (21-25) & 0.90 & $(0.80-1.02)$ & 0.094 & 0.47 & $(0.39-0.56)$ & $<0.001$ \\
\hline Severe (26-35) & 0.74 & $(0.62-0.87)$ & $<0.001$ & 0.41 & $(0.31-0.53)$ & $<0.001$ \\
\hline
\end{tabular}

Boldface indicates statistical significance at the $5 \%$ level (both sides). Estimates are RRRs derived from multinomial logistic regression analyses adjusting for all of generalized trust, PHQ-9, GAD-7, and FCV-19S, plus other explanatory variables (sex, age group, level of education, family members living together, employment, annual household income, bank and saving deposit amount, BMI, pre-existing conditions, and region of residence). All explanatory variables are those at wave 1. Willingness to be vaccinated at wave 3 was the outcome reference group

RRR Relative Risk Ratio, CI Confidence Interval, PHQ-9 Patient Health Questionnaire-9, GAD-7 Generalized Anxiety Disorder -7, FCV-19S Fear of COVID-19 Scale 
How generalized trust is related to vaccine acceptance not only with regard to COVID 19, but also other diseases, has not been sufficiently explored. Although we did not examine the mechanisms linking generalized trust and vaccine acceptance in the present study, previous studies suggest two possible explanations. First, individuals with high levels of generalized trust tend to be more altruistic and intend to contribute to the public health policy of containing COVID-19 [37]. Another possible explanation is that individuals with lower levels of generalized trust may have distrust related to a wide range of issues, including the performance of government $[65,66]$, the safety of the vaccine, and even medical treatments in general [31], leading to vaccine hesitancy. The association between vaccine acceptance and various forms of specific trust, including trust in vaccines, trust in the government, trust in the health system, or in professionals, has already been shown in COVID-19 vaccination $[6,16,20$, $22,26-33]$ and in general $[24,25]$. Several studies, mainly in the social sciences, have demonstrated the positive association between generalized trust and some types of specific trust $[65,66]$. However, to our knowledge, the mediating role of specific trust between generalized trust and vaccine hesitancy has only been explored in Ahorsu et al. [31]. Further studies are recommended to explore how lower levels of generalized trust are associated with COVID-19 vaccine hesitancy.

Regarding the relationship between depression/anxiety and attitudes toward vaccination, the present study showed that participants with depression at waves 1 and 3 were more likely to be undecided regarding vaccination at wave 3 , and participants with generalized anxiety at wave 3 but not at wave 1 were more likely to be unwilling to be vaccinated at wave 3 . There are two things to be considered regarding this finding based on previous studies dealing with similar topics. First, studies in the United States, China, and Germany $[15,17,41]$ found no significant association between depression/anxiety and attitudes toward COVID-19 vaccination, whereas the present study as well as some previous studies [22, 42] found a positive association between psychological distress and vaccine hesitancy. The reason for this disparity is unclear but might be due to the large sample size of our study, which enabled the identification of even minor differences. Another thing to be considered is that studies that address the relationship between depression/anxiety and attitudes toward vaccination other than COVID-19 [43-45] seem to have contradictory results compared to the present study and the two above-mentioned studies on COVID-19 [22, 42], despite the fact that the themes these studies addressed are mostly the same. Chan et al. [43] showed that those who were more anxious were more willing to receive vaccination against $\mathrm{A} / \mathrm{H} 7 \mathrm{~N} 9$ influenza in Hong Kong. Lawrence et al. [44] reported that older primary care patients with mental health diagnoses were more likely to receive vaccination against influenza although the outcome in this study was real vaccine uptake instead of willingness to receive vaccination. Similarly, Mohammed et al. [45] reported that pregnant women with mild depression/anxiety were more likely to receive influenza vaccination during pregnancy although there was no association between depression/ anxiety and willingness to receive vaccination.

Questions still remain as to how mental health factors such as depression and anxiety are associated with vaccine hesitancy in some studies regarding COVID-19, and why the studies on influenza seem to have contradictory results. In addressing these questions, the differences between depression and anxiety may need to be considered. One characteristic of people with depression is that they tend to have difficulty making decisions [67]. The present study's finding that people with depression tend to be undecided about COVID-19 vaccination may be explained by this general indecisiveness. People with generalized anxiety are generally risk averse [68], but what they are anxious about is not predetermined. In the case of vaccination, while there are risks such as side effects from the vaccine, there is also an increased risk of becoming infected with COVID-19 and severely ill by not getting vaccinated. Hence, which of these two risks people with generalized anxiety will prioritize is difficult to determine beforehand without data analysis. The results of the present study suggest that those with moderate and severe level of generalized anxiety at the moment tend to prioritize avoiding the risks associated with getting vaccinated against COVID-19 rather than being infected with COVID-19. Contrastingly, the results of the previous studies [43-45] suggest that those with moderate and severe levels of generalized anxiety tend to prioritize the avoidance of the risks associated with infection (mainly for influenza) over the risks associated with the vaccine. Although we do not have clear answers to explain this seeming difference, there are several possible explanations. First, the subjects in Lawrence et al. [44] were older primary care patients and the subjects in Mohammed et al. [45] were pregnant women who attended an antenatal clinic. By contrast, the subjects in the present study were mostly members of the general public and did not belong in a clinical setting. This difference may explain the variation in results. Second, Chan et al. [43] demonstrated a positive association between anxiety level and willingness to be vaccinated for A/H7N9 influenza based on a population telephone survey, whereas the present study examined the willingness to receive vaccination against COVID-19 based on an online survey. Such differences may explain the seemingly contradictory results. 
Overall, studies exploring the relationship between depression/anxiety and vaccine hesitancy have shown varied results. The diseases that the vaccines are meant to prevent (e.g., COVID-19, seasonal influenza), the methods of measurement of depression/anxiety (e.g., self-administered questionnaire, diagnoses at clinical settings), the coverage of study subjects (e.g., elderly people, pregnant women, general population), as well as the differences in cultural and political backgrounds may be responsible for the varied results in different studies. Further studies are recommended, considering the results of previous studies that are inconsistent with the present study [15, 17, 41, 43-45] and actual vaccination behaviors rather than willingness to receive a vaccine.

The present study found that people with high levels of fear of COVID-19 at waves 1 and 3 were more likely to be willing to receive a vaccine at wave 3 . This finding is consistent with most studies that addressed this issue $[15,22,26,27,41,46-48,54]$. A typical characteristic of fear is that people tend to avoid what they fear. Hence, the most plausible explanation of the negative association between the fear of COVID-19 and vaccine hesitancy is that people with high levels of fear of COVID-19 try to avoid what they fear, namely COVID-19, by getting vaccinated against it. In contrast to most studies including the present one, an older study carried out in Lebanon [46] found no significant association between the willingness to receive the COVID-19 vaccine and the fear of COVID19. The reasons for the difference might be cultural or a difference in sample size.

According to the present study, younger people, women, people with low incomes, and people with lower levels of education are more likely to be undecided or unwilling to be vaccinated. These results are consistent with many previous studies that claim that younger people $[6,7,11,13-15,18-22]$, women $[6,7,9-12,14-16$, 19-23], people with low incomes $[6,11,13-15,18,19$, $21,22]$, and people with lower levels of education $[6,7$, $10,13-16,20,22,23]$ were reluctant to receive a vaccine. Vaccine hesitancy due to younger age and being female may be due to decreased anxiety compared to older males, as there is widely shared information that older people and men are more likely to be seriously affected by COVID-19 infection [69, 70]. Regarding socioeconomic status derived mainly based on income and education, a plausible mediator between low socioeconomic status and vaccine hesitancy is low levels of health protective behaviors due to poor health literacy in people with low socioeconomic status [71-73]. A mediating role of health literacy between socioeconomic status and COVID-19 vaccination hesitancy is suggested in a previous article [72], although it is unclear whether health literacy is the only mediator between them [74]. Therefore, it is safe to state that mechanisms linking such sociodemographic variables and vaccine hesitancy have not been sufficiently explored so far, and further investigation is recommended.

The following limitations must be kept in mind when considering the results of the present study. First, the participants in the present study were not representative of the general population. The present study was based on wave 1 and wave 3 of a longitudinal online survey with the respondents limited to those who responded both at wave 1 and wave 3 . Although wave 1 was designed to reflect sex, age, and place of residence at the prefecture level of the entire Japanese population, there was no random sampling in the extraction of the survey participants. In addition, the number of valid respondents at wave 3 was approximately $70 \%$ of wave 1 . Moreover, the present study was an Internet-based survey; participants were required to have access to the Internet. Possibly reflecting this requirement, almost half of our participants had attended four-year colleges, a percentage greater than Japan's national average. Second, potentially important confounders may not have been adjusted for in the present study. We asked only one question on generalized trust. Specific trusts not covered by the present study may be confounders. Other potential confounders such as place of residence (rural vs urban area) were not incorporated in the present study due to the data constraints. Hence, there remains a possibility that associations between generalized trust (or depression/anxiety, fear of COVID-19) and vaccine hesitancy might be spurious. Third, unlike in many other studies on vaccine hesitancy, COVID-19 vaccination had already started when the question on vaccination was being asked (wave 3), although less than $2 \%$ of Japanese people had been vaccinated by wave 3 . This may have had some impact on attitudes toward vaccine hesitancy. Finally, the present study was an observational study and, thus, cannot demonstrate a causal relationship between the explanatory variables and the outcome variables.

\section{Conclusions}

The present study explored the associations between unwillingness and indecisiveness regarding being vaccinated against COVID-19 with generalized trust, depression, and generalized anxiety. In our findings, low generalized trust, depression, and generalized anxiety were associated with unwillingness and indecisiveness to be vaccinated against COVID-19. In order to determine whether such associations are observed in other countries with different cultures, further epidemiological or sociological studies using global data may be needed. 


\section{Abbreviations}

BMI: Body mass index; COVID-19: Coronavirus disease of 2019; FCV-19S: Fear of COVID-19 Scale; GAD-7: Generalized Anxiety Disorder-7; PHQ-9: Patient Health Questionnaire-9; RIETI: Research Institute of Economy, Trade and Industry; RRR: Relative risk ratio; WVS: World Values Survey.

\section{Supplementary Information}

The online version contains supplementary material available at https://doi. org/10.1186/s12889-021-12479-w.

Additional file 1: Supplementary Table 1. Results of the Fully Adjusted Multinomial Logistic Regression Analyses at wave 3.

\section{Acknowledgements}

We would like to thank the participants.

\section{Authors' contributions}

YS, SH, KD, SO, and SM designed the study; YS drafted the manuscript, carried out the analysis, and interpreted the results; $\mathrm{SH}$ and $\mathrm{SO}$ assisted in drafting and reviewing the manuscript; All authors reviewed the final manuscript and gave their consent.

\section{Funding}

The present study was funded by the RIETI, Japan. The RIETI was involved in the conduct of the study through its contract with NTTCom Online Marketing Solutions Corporation. The RIETI had no role in the study design, data analyses, decision to publish, or preparation of the manuscript.

\section{Availability of data and materials}

The data used in the present study belong to the RIETI and can be obtained from the institute upon reasonable request.

\section{Declarations}

\section{Ethics approval and consent to participate}

Ethical approval was obtained from the ethics committee of Hiramatsu Memorial Hospital (No: 20200925). All individuals who participated in this study provided online informed consent for their participation. All methods were carried out in accordance with relevant guidelines and regulations.

\section{Consent for publication}

Not applicable.

\section{Competing interests}

The authors declare that they have no competing interests.

\section{Author details}

${ }^{1}$ Research Institute of Economy, Trade and Industry, Chiyoda-ku, Kasumigaseki 1-3-1, Tokyo 100-8901, Japan. ${ }^{2}$ United Health Communication Co., Ltd., Chuo-ku, Nihonbashitomizawacho 10-16, Tokyo 103-0006, Japan. ${ }^{3}$ Hiramatsu Memorial Hospital, Chuo-ku Minami 22-jo nishi 14-1-20, Sapporo-shi, Hokkaido 064-8536, Japan. ${ }^{4}$ Department of Laboratory Medicine, The Jikei University School of Medicine, Minato-ku Nishishinbashi 3-25-8, Tokyo 105-8461, Japan. ${ }^{5}$ Department of Psychiatry, Tokyo Dental College Ichikawa General Hospital, Ichikawa-shi, Sugano 5-11-13, Chiba 272-8513, Japan.

Received: 21 July 2021 Accepted: 22 December 2021

Published online: 18 January 2022

\section{References}

1. Ritchie H, Mathieu E, Rodés-Guirao L, Appel C, Giattin C, Ortiz-Ospina E, et al. Coronavirus pandemic (covid-19): Our World in Data; 2020. Available from: https://ourworldindata.org/covid-vaccinations
2. MacDonald NE, SAGE Working Group on Vaccine Hesitancy. Vaccine hesitancy: definition, scope and determinants. Vaccine. 2015;33(34):4161-4. https://doi.org/10.1016/j.vaccine.2015.04.036.

3. Razai MS, Chaudhry UAR, Doerholt K, Bauld L, Majeed A. Covid-19 vaccination hesitancy. BMJ. 2021;373:n1138. https://doi.org/10.1136/bmj. n1138.

4. Sallam M. COVID-19 vaccine hesitancy worldwide: a concise systematic review of vaccine acceptance rates. Vaccines. 2021;9(2):160. https://doi. org/10.3390/vaccines9020160.

5. Volpp KG, Cannuscio CC. Incentives for immunity — strategies for increasing Covid-19 vaccine uptake. N Engl J Med. 2021. https://doi.org/ 10.1056/NEJMp2107719.

6. Paul E, Steptoe A, Fancourt D. Attitudes towards vaccines and intention to vaccinate against COVID-19: implications for public health communications. Lancet Reg Health Eur. 2021;1:100012. https://doi.org/10.1016/j. lanepe.2020.100012

7. Daly M, Robinson E. Willingness to vaccinate against COVID-19 in the U.S.: representative longitudinal evidence from April to October 2020. Am J Prev Med. 2021;60(6):766-73. https://doi.org/10.1016/j.amepre.2021.01. 008.

8. Kourlaba G, Kourkouni E, Maistreli S, Tsopela CG, Molocha NM, Triantafyllou C, et al. Willingness of Greek general population to get a COVID-19 vaccine. Glob Health Res Policy. 2021;6(1):3. https://doi.org/10.1186/ s41256-021-00188-1.

9. Wang C, Han B, Zhao T, Liu H, Liu B, Chen L, et al. Vaccination willingness, vaccine hesitancy, and estimated coverage at the first round of COVID-19 vaccination in China: a national cross-sectional study. Vaccine. 2021;39(21):2833-42. https://doi.org/10.1016/j.vaccine.2021.04.020.

10. Kreps S, Prasad S, Brownstein JS, Hswen Y, Garibaldi BT, Zhang B, et al. Factors associated with US adults' likelihood of accepting COVID-19 vaccination. JAMA Netw Open. 2020;3(10):e2025594. https://doi.org/10.1001/ jamanetworkopen.2020.25594.

11. Edwards B, Biddle N, Gray M, Sollis K. COVID-19 vaccine hesitancy and resistance: correlates in a nationally representative longitudinal survey of the Australian population. PLoS One. 2021;16(3):e0248892. https://doi. org/10.1371/journal.pone.0248892.

12. Yoda T, Katsuyama $\mathrm{H}$. Willingness to receive COVID-19 vaccination in Japan. Vaccines. 2021;9(1):48. https://doi.org/10.3390/vaccines9010048.

13. Williams L, Flowers P, McLeod J, Young D, Rollins L. The catalyst project team. Social patterning and stability of intention to accept a COVID-19 vaccine in Scotland: will those most at risk accept a vaccine? Vaccines. 2021:9(1):17. https://doi.org/10.3390/vaccines9010017.

14. Robertson E, Reeve KS, Niedzwiedz CL, Moore J, Blake M, Green M, et al. Predictors of COVID-19 vaccine hesitancy in the UK household longitudinal study. Brain Behav Immun. 2021;94:41-50. https://doi.org/10.1016/j. bbi.2021.03.008.

15. Killgore WDS, Cloonan SA, Taylor EC, Dailey NS. The COVID-19 vaccine is here-now who is willing to get it? Vaccines (Basel). 2021;9(4):339. https://doi.org/10.3390/vaccines9040339.

16. Petravić L, Arh R, Gabrovec T, Jazbec L, Rupčić N, Starešinič N, et al. Factors affecting attitudes towards COVID-19 vaccination: an online survey in Slovenia. Vaccines. 2021;9(3):247. https://doi.org/10.3390/vaccines90 30247.

17. Bai W, Cai H, Liu S, Liu H, Qi H, Chen X, et al. Attitudes toward COVID-19 vaccines in Chinese college students. Int J Biol Sci. 2021;17(6):1469-75. https://doi.org/10.7150/ijbs.58835.

18. Ditekemena JD, Nkamba DM, Mutwadi A, Mavoko HM, Siewe Fodjo JN, Luhata C, et al. COVID-19 vaccine acceptance in the Democratic Republic of Congo: a cross-sectional survey. Vaccines (Basel). 2021;9(2):153. https:// doi.org/10.3390/vaccines9020153.

19. Kadoya Y, Watanapongvanich S, Yuktadatta P, Putthinun P, Lartey ST, Khan MSR. Willing or hesitant? A socioeconomic study on the potential acceptance of COVID-19 vaccine in Japan. Int J Environ Res Public Health. 2021;18(9):4864. https://doi.org/10.3390/ijerph18094864.

20. Soares P, Rocha JV, Moniz M, Gama A, Laires PA, Pedro AR, et al. Factors associated with COVID-19 vaccine hesitancy. Vaccines (Basel). 2021;9(3):300. https://doi.org/10.3390/vaccines9030300.

21. Machida M, Nakamura I, Kojima T, Saito R, Nakaya T, Hanibuchi T, et al. Acceptance of a COVID-19 vaccine in Japan during the COVID-19 pandemic. Vaccines. 2021;9(3):210. https://doi.org/10.3390/vaccines9030210. 
22. Okubo R, Yoshioka T, Ohfuji S, Matsuo T, Tabuchi T. COVID-19 vaccine hesitancy and its associated factors in Japan. Vaccines. 2021;9(6):662. https:// doi.org/10.3390/vaccines9060662.

23. Ogilvie GS, Gordon S, Smith LW, Albert A, Racey CS, Booth A, et al. Intention to receive a COVID-19 vaccine: results from a population-based survey in Canada. BMC Public Health. 2021;21 (1):1017. https://doi.org/10. 1186/s12889-021-11098-9.

24. Truong J, Bakshi S, Wasim A, Ahmad M, Majid U. What factors promote vaccine hesitancy or acceptance during pandemics? A systematic review and thematic analysis. Health Promot Int. 2021. https://doi.org/10.1093/ heapro/daab105.

25. Larson HJ, Clarke RM, Jarrett C, Eckersberger E, Levine Z, Schulz WS, et al. Measuring trust in vaccination: a systematic review. Hum Vaccin Immunother. 2018;14(7):1599-609. https://doi.org/10.1080/21645515.2018. 1459252.

26. Hwang SE, Kim W-H, Heo J. Socio-demographic, psychological, and experiential predictors of COVID-19 vaccine hesitancy in South Korea, October-December 2020. Hum Vaccin Immunother. 2021:1-8. https://doi. org/10.1080/21645515.2021.1983389.

27. Willis DE, Andersen JA, Bryant-Moore K, Selig JP, Long CR, Felix HC, et al. Covid-19 vaccine hesitancy: race/ethnicity, trust, and fear. Clin Transl Sci. 2021. https://doi.org/10.1111/cts.13077 Epub 2021/07/03. 34213073.

28. Blanchi S, Torreggiani M, Chatrenet A, Fois A, Mazé B, Njandjo L, et al. Covid-19 vaccine hesitancy in patients on dialysis in Italy and France. Kidney Int Rep. 2021;6(11):2763-74. https://doi.org/10.1016/j.ekir.2021.08. 030.

29. Lazarus JV, Ratzan SC, Palayew A, Gostin LO, Larson HJ, Rabin K, et al. A global survey of potential acceptance of a COVID-19 vaccine. Nat Med. 2021;27(2):225-8. https://doi.org/10.1038/s41591-020-1124-9 Epub 2020/10/22

30. Rocha ICN. Employing medical anthropology approach as an additional public health strategy in promoting COVID-19 vaccine acceptance in Bhutan. Int J Health Plann Manag. 2021;36(5):1943-6. https://doi.org/10. 1002/hpm.3191.

31. Ahorsu DK, Lin C-Y, Yahaghai R, Alimoradi Z, Broström A, Griffiths MD, et al. The mediational role of trust in the healthcare system in the association between generalized trust and willingness to get COVID-19 vaccination in Iran. Hum Vaccines Immunother. 2021:1-8. https://doi.org/10.1080/ 21645515.2021.1993689.

32. Al-Mohaithef M, Padhi BK, Ennaceur S. Socio-demographics correlate of COVID-19 vaccine hesitancy during the second wave of COVID-19 pandemic: a cross-sectional web-based survey in Saudi Arabia. Front Public Health. 2021;9:698106. https://doi.org/10.3389/fpubh.2021.698106 34249851.

33. Huynh HP, Zsila Á, Martinez-Berman L. Psychosocial predictors of intention to vaccinate against the coronavirus (COVID-19). Behav Med. 2021:1-15. https://doi.org/10.1080/08964289.2021.1990006.

34. Miller AS, Mitamura T. Are surveys on trust trustworthy? Soc Psychol Q. 2003;66(1):62-70. https://doi.org/10.2307/3090141.

35. Rothstein B, Stolle D. The state and social capital: an institutional theory of generalized trust. Comp Polit. 2008;40(4):441-59. https://doi.org/10.5129/ $001041508 \times 12911362383354$.

36. Frank K, Arim R. Canadians' willingness to get a COVID-19 vaccine when one becomes available: what role does trust play? Statistics Canada catalogue no. 45280001. Ottawa: Statistics Canada; 2020

37. Rönnerstrand B. Social capital and immunisation against the 2009 A(H1N1) pandemic in Sweden. Scand J Public Health. 2013;41(8):853-9. https://doi.org/10.1177/1403494813494975.

38. Rönnerstrand B. Social capital and immunization against the 2009 A(H1N1) pandemic in the American States. Public Health. 2014;128(8):709-15. https://doi.org/10.1016/j.puhe.2014.05.015.

39. Freimuth VS, Jamison AM, An J, Hancock GR, Quinn SC. Determinants of trust in the flu vaccine for African Americans and Whites. Soc Sci Med. 2017:193:70-9. https://doi.org/10.1016/j.socscimed.2017.10.001.

40. Mazereel V, Van Assche K, Detraux J, De Hert M. COVID-19 vaccination for people with severe mental illness: why, what, and how? Lancet Psychiatry. 2021;8(5):444-50. https://doi.org/10.1016/S2215-0366(20)30564-2.

41. Bendau A, Plag J, Petzold MB, Ströhle A. COVID-19 vaccine hesitancy and related fears and anxiety. Int Immunopharmacol. 2021;97:107724. https:// doi.org/10.1016/j.intimp.2021.107724.
42. Xu Y, Zhang R, Zhou Z, Fan J, Liang J, Cai L, et al. Parental psychological distress and attitudes towards COVID-19 vaccination: a cross-sectional survey in Shenzhen, China. J Affect Disord. 2021;292:552-8. https://doi. org/10.1016/j.jad.2021.06.003.

43. Chan EY-Y, Cheng CK-Y, Tam GC-H, Huang Z, Lee PY. Willingness of future A/H7N9 influenza vaccine uptake: a cross-sectional study of Hong Kong community. Vaccine. 2015;33(38):4737-40. https://doi.org/10.1016/j.vacci ne.2015.07.046

44. Lawrence T, Zubatsky M, Meyer D. The association between mental health diagnoses and influenza vaccine receipt among older primary care patients. Psychol Health Med. 2020;25(9):1083-93. https://doi.org/10. 1080/13548506.2020.1717557.

45. Mohammed H, Roberts CT, Grzeskowiak LE, Giles L, Leemaqz S, Dalton J, et al. Psychosocial determinants of pertussis and influenza vaccine uptake in pregnant women: a prospective study. Vaccine. 2020;38(17):3358-68. https://doi.org/10.1016/j.vaccine.2020.02.020.

46. Kasrine Al Halabi C, Obeid S, Sacre H, Akel M, Hallit R, Salameh P, et al. Attitudes of Lebanese adults regarding COVID-19 vaccination. BMC Public Health. 2021;21 (1):998. https://doi.org/10.1186/s12889-021-10902-w.

47. Nazlı ŞB, Yığman F, Sevindik M, Deniz Özturan D. Psychological factors affecting COVID-19 vaccine hesitancy. Iran J Med Sci. 2021:1-10. https:// doi.org/10.1007/s11845-021-02640-0.

48. McElfish PA, Willis DE, Shah SK, Bryant-Moore K, Rojo MO, Selig JP. Sociodemographic determinants of COVID-19 vaccine hesitancy, fear of infection, and protection self-efficacy. J Prim Care Community Health. 2021;12:21501327211040746. https://doi.org/10.1177/215013272110407 46. 34427126.

49. Kwon Y, Cho H-Y, Lee Y-K, Bae G-R, Lee S-G. Relationship between intention of novel influenza $\mathrm{A}(\mathrm{H} 1 \mathrm{~N} 1)$ vaccination and vaccination coverage rate. Vaccine. 2010;29(2):161-5. https://doi.org/10.1016/j.vaccine.2010.10. 063.

50. Nguyen TTM, Lafond KE, Nguyen TX, Tran PD, Nguyen HM, Ha VTC, et al. Acceptability of seasonal influenza vaccines among health care workers in Vietnam in 2017. Vaccine. 2020;38(8):2045-50. https://doi.org/10. 1016/j.vaccine.2019.12.047.

51. Patil SS, Patil SR, Ganla A, Durgawale PM. Knowledge and awareness about cervical cancer and human papilloma virus (HPV) vaccine among nursing students. J Crit Rev. 2020;7(12):384-93. https://doi.org/10.31838/ jcr.07.12.73.

52. Anraad C, Lehmann BA, Visser O, van Empelen P, Paulussen TGW, Ruiter RAC, et al. Social-psychological determinants of maternal pertussis vaccination acceptance during pregnancy among women in the Netherlands. Vaccine. 2020;38(40):6254-66. https://doi.org/10.1016/j.vaccine.2020.07. 047.

53. Bhanu C, Gopal DP, Walters K, Chaudhry UAR. Vaccination uptake amongst older adults from minority ethnic backgrounds: a systematic review. PLoS Med. 2021;18(11):e1003826. https://doi.org/10.1371/journal. pmed. 1003826.

54. Pelegrín-Borondo J, Arias-Oliva M, Almahameed AA, Prado Román M. Covid-19 vaccines: a model of acceptance behavior in the healthcare sector. Eur Res Manag Bus Econ. 2021;27(3):100171. https://doi.org/10.1016/j. iedeen.2021.100171.

55. Inglehart R, Haerpfer C, Moreno A, Welzel C, Kizilova K, Diez-Medrano J, et al. World values survey: round six - country-pooled datafile version. Madrid: JD Systems Institute; 2014. https://www.worldvaluessurvey.org/ WVSDocumentationWV6.jsp

56. Johnson ND, Mislin A. How much should we trust the world values survey trust question? Econ Lett. 2012;116(2):210-2. https://doi.org/10. 1016/j.econlet.2012.02.010.

57. Knack S, Keefer P. Does social capital have an economic payoff? A crosscountry investigation. Q J Econ. 1997;112(4):1251-88. https://doi.org/10. 1162/003355300555475.

58. Kroenke K, Spitzer RL, Williams JB. The PHQ-9: validity of a brief depression severity measure. J Gen Intern Med. 2001;16(9):606-13. https://doi.org/ 10.1046/j.1525-1497.2001.016009606.x.

59. Muramatsu K, Kamijima K, Yoshida M, Otsubo T, Miyaoka H, Muramatsu Y, et al. The patient health questionnaire, Japanese version: validity according to the mini-international neuropsychiatric interview-plus. Psychol Rep. 2007;101(3):952-60. https://doi.org/10.2466/pr0.101.3.952-960.

60. Muramatsu K, Miyaoka H, Kamijima K, Muramatsu Y, Tanaka Y, Hosaka $\mathrm{M}$, et al. Performance of the Japanese version of the Patient Health 
Questionnaire-9 (J-PHQ-9) for depression in primary care. Gen Hosp Psychiatry. 2018;52:64-9. https://doi.org/10.1016/j.genhosppsych.2018. 03.007.

61. Spitzer RL, Kroenke K, Williams JBW, Löwe B. A brief measure for assessing generalized anxiety disorder: the GAD-7. Arch Intern Med. 2006;166(10):1092-7. https://doi.org/10.1001/archinte.166.10.1092.

62. Muramatsu K, Muramatsu Y, Miyaoka H, Fuse K, Yoshimine F, Hosaka M, et al., editors. Validation and utility of a Japanese version of the GAD-7. PANMINERVA MEDICA 20th World Congress on Pyschosomatic Medicine Abstracts Book; 2009.

63. Ahorsu DK, Lin CY, Imani V, Saffari M, Griffiths MD, Pakpour AH. The fear of COVID-19 scale: development and initial validation. Int J Ment Health Addict. 2020:1-9. https://doi.org/10.1007/s11469-020-00270-8.

64. Masuyama A, Shinkawa H, Kubo T. Validation and psychometric properties of the Japanese version of the fear of COVID-19 scale among adolescents. Int J Ment Health Addict. 2020:1-11. https://doi.org/10.1007/ s11469-020-00368-z.

65. Park HB. Is trust in government a short-term strategic value or a longterm democratic value? A case study of three Nordic countries and three East Asian nations. Int Rev Public Admin. 2015;20(3):273-86. https://doi. org/10.1080/12294659.2015.1067952.

66. Zmerli S, Newton K. Social trust and attitudes toward democracy. Public Opin Q. 2008;72(4):706-24. https://doi.org/10.1093/poq/nfn054.

67. Di Schiena R, Luminet O, Chang B, Philippot P. Why are depressive individuals indecisive? Different modes of rumination account for indecision in non-clinical depression. Cognit Ther Res. 2013;37(4):713-24. https:// doi.org/10.1007/s10608-012-9517-9.

68. Hartley CA, Phelps EA. Anxiety and decision-making. Biol Psychiatry. 2012;72(2):113-8. https://doi.org/10.1016/j.biopsych.2011.12.027.

69. Liu K, Chen Y, Lin R, Han K. Clinical features of COVID-19 in elderly patients: a comparison with young and middle-aged patients. J Infect. 2020;80(6):e14-e8. https://doi.org/10.1016/j.jinf.2020.03.005.

70. Peckham H, de Gruijter NM, Raine C, Radziszewska A, Ciurtin C, Wedderburn LR, et al. Male sex identified by global COVID-19 meta-analysis as a risk factor for death and ITU admission. Nat Commun. 2020;11(1):6317. https://doi.org/10.1038/s41467-020-19741-6.

71. Coburn D, Pope CR. Socioeconomic status and preventive health behaviour. J Health Soc Behav. 1974;15(2):67-78. https://doi.org/10.2307/21371 86.

72. Kricorian K, Civen R, Equils O. Covid-19 vaccine hesitancy: misinformation and perceptions of vaccine safety. Hum Vaccin Immunother. 2021:1-8. https://doi.org/10.1080/21645515.2021.1950504.

73. Tokuda Y, Okubo T, Yanai H, Doba N, Paasche-Orlow MK. Development and validation of a 15-item Japanese health knowledge test. J Epidemiol. 2010;20(4):319-28. https://doi.org/10.2188/jea.JE20090096.

74. Howard DH, Sentell T, Gazmararian JA. Impact of health literacy on socioeconomic and racial differences in health in an elderly population. J Gen Intern Med. 2006;21(8):857-61. https://doi.org/10.1111/j.1525-1497.2006. 00530.x.

\section{Publisher's Note}

Springer Nature remains neutral with regard to jurisdictional claims in published maps and institutional affiliations.

Ready to submit your research? Choose BMC and benefit from:

- fast, convenient online submission

- thorough peer review by experienced researchers in your field

- rapid publication on acceptance

- support for research data, including large and complex data types

- gold Open Access which fosters wider collaboration and increased citations

- maximum visibility for your research: over $100 \mathrm{M}$ website views per year

At BMC, research is always in progress.

Learn more biomedcentral.com/submissions 